\title{
Reformen zur Entlastung geringer Einkommen und ihre Auswirkungen auf die Beschäftigung
}

\begin{abstract}
Die Corona-Krise hat die Wirtschaft zwar belastet, doch bietet sie auch die Chance, durch Reformen des Sozialversicherungssystems den Aufbau guter Beschäftigung zu fördern. Auf Basis eines makroökonomischen Modells untersucht der Beitrag zwei Reformvarianten, die den sozialrechtlichen Sonderstatus des Minijobs abschaffen und zudem Erwerbspersonen mit niedrigem Einkommen entlasten. Die Ergebnisse der Simulationsanalyse zeigen, dass eine Reform zur Abschaffung der Minijob-Regelung bei gleichzeitiger Entlastung der unteren Einkommensgruppen (bis 1.800 Euro brutto im Monat) viele Gewinner:innen hat. Die Reform hätte positive Effekte für Wirtschaft, Gesellschaft und öffentliche Finanzen.
\end{abstract}

Die Corona-Krise hat Wirtschaft und Gesellschaft hart getroffen, doch es gibt auch Grund zur Hoffnung. Die Krise bietet eine Chance, strukturelle Reformen voranzutreiben und gestärkt aus der Krise hervorzugehen. Eine wichtige Reform betrifft das Sozialversicherungssystem.

Deutschland hat ein relativ gut ausgebautes System der gesetzlichen Arbeitslosen-, Kranken- und Rentenversicherung. Das hat viele Vorteile, aber auch den Nachteil, dass niedrige Erwerbseinkommen relativ stark durch Sozialversicherungsbeiträge belastet werden. Die hohe Abgabenbelastung der niedrigen Erwerbseinkommen im Zusammenspiel mit den geringen Stundenlöhnen im Niedriglohnbereich haben zur Folge, dass sich Arbeit für viele Erwerbspersonen in Deutschland kaum lohnt.

Dieses Problem hat auch die Bundesregierung erkannt, und seit 1. Juli 2019 wurden die Sozialversicherungsbeiträge für geringe Erwerbseinkommen gesenkt. Konkret wurde die bisherige Midi-Job-Gleitzone für monatliche Bruttoeinkommen zwischen 450 Euro (Minijob) und 850 Euro durch einen erweiterten Übergangsbereich von 450 Euro bis 1.300 Euro abgelöst.

(C) Der/die Autor:in 2021. Open Access: Dieser Artikel wird unter der Creative Commons Namensnennung 4.0 International Lizenz veröffentlicht (creativecommons.org/licenses/by/4.0/deed.de).

Open Access wird durch die ZBW - Leibniz-Informationszentrum Wirtschaft gefördert.

* Dieser Text stellt eine Zusammenfassung der im Juni 2021 veröffentlichten Studie „Raus aus der Minijobfalle - Reformen zur Entlastung geringer Einkommen und ihre Auswirkungen auf Beschäftigung, Wachstum und Verteilung sowie die öffentlichen Finanzen" (Krebs und Scheffel, 2021) dar, die im Auftrag der Bertelsmann Stiftung entstanden ist.
Die Reform vom 1. Juli 2019 ist ein Schritt in die richtige Richtung, doch greift sie aus zwei Gründen zu kurz. Erstens belässt die Reform den sozialrechtlichen Sonderstatus des Minijobs und subventioniert somit Beschäftigungsverhältnisse mit unterdurchschnittlicher Produktivität. Zudem ist die mangelnde soziale Absicherung von Minijobs ein bekanntes Problem, das in der Krise besonders deutlich wurde.

Zweitens entlastet die Reform nicht die Einkommen über 1.300 Euro und hat somit (fast) keine positiven Auswirkungen auf die Vollzeitbeschäftigung und gut bezahlte Teil-

Prof. Tom Krebs, Ph.D., ist Professor für Makroökonomik und Wirtschaftspolitik an der Universität Mannheim.

Dr. Martin Scheffel ist Lecturer Above the Bar an der National University of Ireland, Galway.

Manuela Barišić ist Senior Projektmanagerin im arbeitsmarkt- und sozialpolitischen Projekt „Beschäftigung im Wandel“ der Bertelsmann Stiftung.

Valentina Sara Consiglio ist Projektmanagerin im arbeitsmarkt- und sozialpolitischen Projekt „Beschäftigung im Wandel“ der Bertelsmann Stiftung. 
zeitbeschäftigung. Die aktuelle Krise bzw. die Zeit nach der Pandemie bietet die Chance, aufbauend auf der Reform vom 1. Juli 2019 eine größere Reform der Sozialversicherungsbeiträge zu implementieren, die das Sozialversicherungssystem in diese zwei Richtungen weiterentwickelt und somit einen wichtigen Beitrag zum Aufbau guter Beschäftigung leistet.

\section{Reformen zur Entlastung niedriger Einkommen und Abschaffung des Sonderstatus des Minijobs}

Diese Überlegungen bilden den Hintergrund der vorliegenden Analyse. Es werden zwei Reformen untersucht, die beide den sozialrechtlichen Sonderstatus des Minijobs abschaffen und gleichzeitig viele Erwerbspersonen mit niedrigem Einkommen entlasten. Dabei schafft nur die zweite Reformvariante eine breite Entlastung der unteren Einkommen. Die zwei Reformvarianten sind (vgl. auch Abbildung 1): ${ }^{1}$

- Reformvariante 1 schafft den Sonderstatus des Minijobs ab, indem sie eine linear ansteigende Durchschnittsbelastung von $0 \%$ bei einem Bruttoeinkommen von 0 Euro auf 20,2\% am oberen Ende des aktuellen Übergangsbereichs einführt.

- Reformvariante 2 schafft ebenfalls den Sonderstatus des Minijobs ab und entlastet zusätzlich geringe Einkommen im Niedriglohnbereich. Konkret führt die zweite Reformvariante wie Reformvariante 1 eine linear ansteigende Durchschnittsbelastung ein, weitet aber gleichzeitig die Obergrenze des Übergangsbereichs von derzeit 1.300 Euro auf 1.800 Euro aus und entlastet somit zusätzlich vollzeitbeschäftigte Erwerbspersonen im Niedriglohnbereich bis 1.800 Euro.

\section{Der zugrundeliegende Modellrahmen}

Die Evaluation der unterschiedlichen Reformvarianten basiert auf einem mikroökonomisch fundierten makroökonomischen Modell der deutschen Volkswirtschaft. Der Fokus der Analyse liegt auf der Entwicklung des Produktionspotenzials - kurzfristige keynesianische Nachfrageeffekte werden vernachlässigt. Das Modell erlaubt eine empirisch fundierte dynamische Analyse wirtschaftspolitischer Maßnahmen, die neben den direkten Effekten auch die indirekten Effekte berücksichtigt, die durch Verhaltensanpassungen der privaten Haushalte und Unternehmen sowie Wechselwirkungen zwischen Arbeits-, Kapital- und Gütermärkten verursacht werden. Die im Modellrahmen ab-

1 Die Auswirkungen der Reform vom 1. Juli 2019 werden auch in Krebs und Scheffel (2021) untersucht, aber hier nicht weiter besprochen.

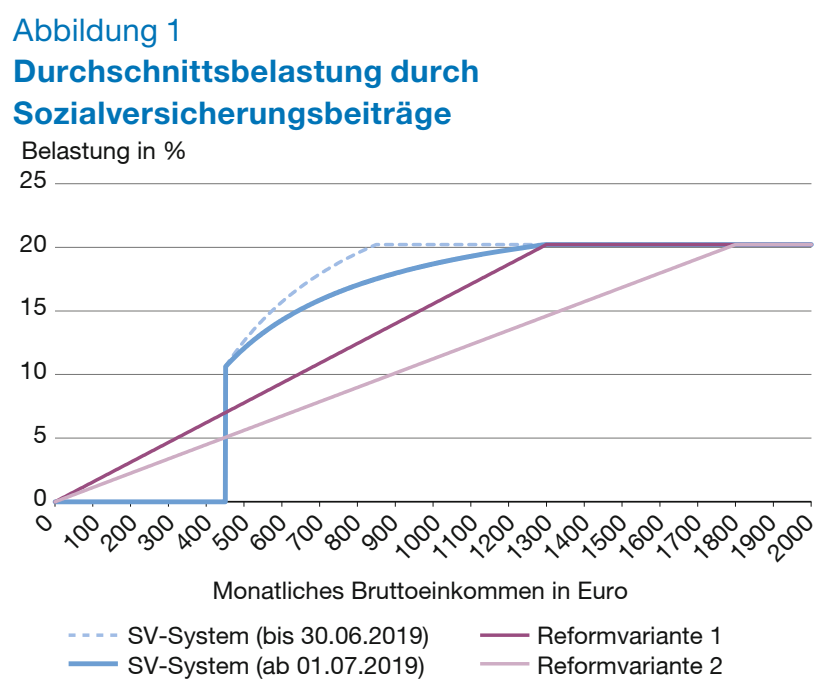

Quelle: eigene Berechnung.

gebildeten Friktionen und Anreizsysteme ${ }^{2}$ führen zu einer Verzerrung privater Investitionsanreize und bedingen die Notwendigkeit öffentlicher Investitionen.

\section{Die zugrundeliegenden Wirkungskanäle}

Das Modell bildet die zentralen Wirkungskanäle ab, sodass kausale Zusammenhänge konsistent analysiert werden können. Die Entlastung geringer und mittlerer Einkommen durch eine Reform der Sozialversicherungsbeiträge hat dabei direkte und indirekte Effekte. Die direkten Effekte sind eine Folge von Verhaltensänderungen von Erwerbspersonen mit geringen und mittleren Einkommen. Jede der hier betrachteten Reformen führt zu einer durchschnittlichen Entlastung des Arbeitseinkommens und steigert somit für arbeitslose Erwerbspersonen den Anreiz zur Stellensuche. Darüber hinaus ergeben sich je nach Reform zusätzliche Anreizeffekte.

Insbesondere motivieren die Reformvarianten 1 und 2 geringfügig Beschäftigte, ihr Arbeitsangebot auszuweiten und nach einer Teilzeit- oder Vollzeitbeschäftigung zu suchen: Das Problem der „Minijobfalle“ wird abgeschwächt. Mit einem Wechsel aus geringfügiger Beschäftigung in

2 Im Modell sind private Haushalte unvollständig gegen Einkommensrisiken versichert. Zudem steigen die Steuer- und Sozialabgaben der privaten Haushalte mit dem geleisteten Arbeitsvolumen. Diese Faktoren hemmen Ausweitungen des Arbeitsangebots. Außerdem behindern Suchfriktionen auf den Arbeitsmärkten die optimale Allokation der Produktionsfaktoren. Ökonomische Externalitäten, die zum Beispiel durch ein gemeinschaftlich finanziertes Sozialversicherungssystem entstehen, führen zu unterschiedlichen privaten und sozialen Renditen, sodass Entlastungen geringer und mittlerer Einkommen durchaus gesamtwirtschaftliche Effekte haben und zu einer verbesserten Allokation der Produktionsfaktoren führen. 
Teilzeit- oder Vollzeitbeschäftigung geht typischerweise ein Anstieg der Arbeitsproduktivität und des Lohns einher, der wiederum zusätzliche Wachstumsimpulse setzt. ${ }^{3}$ Zeitgleich kann jedoch auch ein gegenläufiger Effekt am oberen Ende der Gleitzone entstehen, wenn die Entlastung der Teilzeitbeschäftigung größer ist als die Entlastung der Vollzeitbeschäftigung. In solchen Fällen reduziert sich der Anreiz für Teilzeitbeschäftigte, ihre Arbeitszeit auszuweiten und nach einer Vollzeitbeschäftigung zu suchen. Infolgedessen steigt die Teilzeitbeschäftigung an, während die Vollzeitbeschäftigung sinkt. In diesem Sinne können die hier vorgeschlagenen Reformen 1 und 2 Teilzeitarbeit unnötig verstärken. Dieser Teilzeiteffekt reduziert die durchschnittliche Produktivität und Entlohnung, weil Teilzeitarbeit typischerweise weniger produktiv ist als Vollzeitarbeit. ${ }^{4}$

Neben den direkten Effekten gibt es eine Reihe indirekter Effekte, die durch die Verhaltensanpassungen der privaten Haushalte und Unternehmen und die Wechselwirkungen zwischen Arbeits-, Kapital- und Gütermärkten entstehen. Beispielweise stimulieren Produktivitätsgewinne die Nachfrage der Unternehmen nach Arbeit und Kapital, sodass Beschäftigung, Stundenlöhne und private Investitionen zunehmen. Diese Zuwächse bei Beschäftigung, Produktivität und Löhnen vermehren mit zeitlicher Verzögerung die Einnahmen der öffentlichen Hand in Form von Steuern und Sozialabgaben, sodass die anfänglichen Rückgänge kompensiert werden, während gleichzeitig die Ausgaben für Sozialleistungen sinken.

Bei der Interpretation der Ergebnisse ist zu beachten, dass die Analyse nur einen Teil der möglichen Anpassungsreaktionen der Haushalte und Unternehmen berücksichtigt. Insbesondere werden die folgenden drei Transmissionskanäle vernachlässigt.

Erstens wird die Zahl der Arbeitsstunden innerhalb einer Beschäftigungsart (Vollzeitbeschäftigung, Teilzeitbeschäftigung, Minijob) in der Modellanalyse konstant gehalten, sodass etwaige marginale Anpassungen der Arbeitszeit nicht erfasst werden. Zweitens geht die vorliegende Untersuchung von der Annahme einer konstanten Suchintensität der Unternehmen aus. Diese Annahme impliziert, dass sich die positiven Effekte einer Steigerung der Unternehmensproduktivität nicht auf die Anreize der Unternehmen auswirken, Produktion und Beschäftigung auszuweiten. Folglich werden durch verstärkte Suchaktivitäten verursachte positive Impulse auf die Raten des

3 Der beschriebene Zusammenhang zwischen Beschäftigungsart und Produktivität bzw. Lohn ist empirisch belegt; die einschlägige Literatur wird in Krebs und Scheffel (2015) diskutiert. Der dieser Analyse zugrundeliegende Modellrahmen ist so kalibriert, dass das Modell die empirisch geschätzten Lohnunterschiede akkurat abbildet.

4 Auch dieser Effekt ist empirisch belegt (Krebs und Scheffel, 2015).
Übergangs aus Arbeitslosigkeit in Beschäftigung bzw. aus bestehender Beschäftigung in umfangreichere Beschäftigungsverhältnisse in der Analyse vernachlässigt. Drittens werden kurzfristige Effekte vernachlässigt, die durch den keynesianischen Nachfragekanal erzeugt werden und zu einer vorübergehenden Stimulation der gesamtwirtschaftlichen Produktion und damit verbunden zu einer Beschäftigungsausweitung führen.

Da der vorliegende Modellrahmen diese drei Wirkmechanismen vernachlässigt, werden die positiven Beschäftigungseffekte der Reformen tendenziell unterschätzt.

\section{Die Analyse und die zentralen Ergebnisse der Modellsimulation}

Die direkten Effekte der beiden Reformvarianten auf das Nettoeinkommen der privaten Haushalte berechnen sich unmittelbar aus den Daten (vgl. Tabelle 1 und 2): Reformvariante 1 entlastet $13,3 \%$ und belastet $8,1 \%$ der Erwerbspersonen. Die direkten fiskalischen Kosten sind relativ gering, weil sich Einnahmenausfälle und Mehreinnahmen ungefähr die Waage halten. Reformvariante 2 hingegen entlastet 26,1\% der Erwerbspersonen zum Teil erheblich und belastet Erwerbspersonen in Minijobs etwas weniger als Reformvariante 1. Die direkten fiskalischen Kosten der Reformvariante 2 belaufen sich auf 4,54 Mrd. Euro im ersten Jahr. ${ }^{5}$ Diesen Kosten stehen langsam steigende fiskalische Nettomehreinnahmen gegenüber, die bis 2050 jährlich 2,21 Mrd. Euro erreichen.

Die dynamischen Effekte der Reformen werden im Modellrahmen simuliert. Die wesentlichen Ergebnisse können wie folgt zusammengefasst werden:

Reformvariante 1 führt langfristig zu einem moderaten Rückgang der Arbeitslosigkeit um 8.900 Erwerbspersonen. Wie die Reform vom 1. Juli 2019 steigert diese Variante hauptsächlich die Teilzeitbeschäftigung und verstärkt somit deren Verfestigung. Im Gegensatz zur Reform vom 1. Juli 2019 verringert Reformvariante 1 jedoch die geringfügige Beschäftigung und hilft in diesem Sinne bei der Beseitigung der Minijobfalle. Reformvariante 1 hat einen moderat positiven Effekt auf das Wirtschaftswachstum und verändert die Armut bzw. Ungleichheit nur geringfügig. Sie erzeugt zusätzliche Einnahmen für die öffentliche Hand, die im Jahr 2033 erstmals die direkten fiskalischen Kosten übersteigen (vgl. Tabelle 1).

Im Gegensatz hierzu sind die positiven Effekte der Reformvariante 2 auf Arbeitsmarkt und Wachstum deutlich größer.

5 Die Modellsimulation startet dabei in 2020 und damit nach der Reform vom 1. Juli 2019 
Tabelle 1

Übersicht der Simulationsergebnisse - Reformvariante 1

\begin{tabular}{|c|c|c|c|c|c|c|c|c|c|}
\hline & \multicolumn{3}{|c|}{$\begin{array}{c}\text { Alle } \\
\text { Erwerbspersonen }\end{array}$} & \multicolumn{3}{|c|}{$\begin{array}{l}\text { Geringqualifizierte } \\
\text { Erwerbspersonen }\end{array}$} & \multicolumn{3}{|c|}{$\begin{array}{l}\text { Alleinerziehende } \\
\text { Erwerbspersonen }\end{array}$} \\
\hline & 2020 & 2030 & 2050 & 2020 & 2030 & 2050 & 2020 & 2030 & 2050 \\
\hline BIP (in Mrd. Euro) & 0 & $+1,10$ & $+2,81$ & & & & & & \\
\hline Fiskalische Nettomehreinnahmen (in Mrd. Euro) & $-0,56$ & $-0,10$ & $+0,59$ & & & & & & \\
\hline \multicolumn{10}{|l|}{ Beschäftigung (absolut) } \\
\hline arbeitslos & 0 & -8.200 & -8.900 & 0 & -4.400 & -4.900 & 0 & -1.500 & -2.000 \\
\hline geringfügig beschäftigt & 0 & -36.000 & -35.900 & 0 & -2.500 & -2.400 & 0 & -5.900 & -5.900 \\
\hline teilzeitbeschäftigt & 0 & +40.900 & +41.300 & 0 & +5.300 & +5.700 & 0 & +6.500 & +6.900 \\
\hline vollzeitbeschäftigt & 0 & +3.300 & +3.500 & 0 & +1.500 & +1.600 & 0 & +900 & +1.000 \\
\hline Beschäftigung (VZ-Äquivalent) & 0 & +14.800 & +15.200 & 0 & +3.500 & +3.900 & 0 & +2.700 & +3.000 \\
\hline Armutsgefährdungsquote (in Prozentpunkten) & 0 & $-0,03$ & $-0,03$ & 0 & $-0,93$ & $-0,93$ & 0 & $-0,03$ & $-0,04$ \\
\hline Median der Armutsgefährdung (in Euro) & 0 & $+0,46$ & $+0,46$ & 0 & $+5,78$ & $+5,81$ & 0 & $+2,99$ & $+3,30$ \\
\hline Armutsquote (in Prozentpunkten) & 0 & 0 & 0 & 0 & $+0,18$ & $+0,17$ & 0 & $-0,03$ & $-0,03$ \\
\hline
\end{tabular}

Anmerkungen: Ergebnis der Modellsimulation. Die Werte geben die jeweiligen Veränderungen gegenüber der Situation seit der Reform vom 1. Juli 2019 wieder. Die Armutsgefährdungsquote ist der Anteil der Erwerbspersonen, die nach Transferzahlungen über weniger als $60 \%$ des Median-Nettoeinkommens verfügen (2019: 15,9\%). Der Median der Armutsgefährdung ist das Median-Nettoeinkommen der armutsgefährdeten Erwerbspersonen. Die Armutsquote ist der Anteil der Erwerbspersonen, die ein Nettoeinkommen unterhalb der Grundsicherung beziehen (2019: 9,6\%).

Quelle: eigene Berechnung.

Konkret reduziert die Reform langfristig die Arbeitslosigkeit um 92.600 Erwerbspersonen. Zudem steigen sowohl die Teilzeit- als auch die Vollzeitbeschäftigung erheblich an, während die geringfügige Beschäftigung stark zurückgeht, sodass insgesamt ein Zuwachs der Beschäftigung um 68.900 vollzeitäquivalente Stellen zu erwarten ist.

In diesem Sinne schafft diese Reform gute Arbeit, die sozial abgesichert und fairer entlohnt ist. Geringqualifizierte und alleinerziehende Erwerbspersonen profitieren von Reformvariante 2 besonders stark, und der Anstieg der Beschäftigung in diesen beiden Gruppen ist überproportional hoch (vgl. Tabelle 2). Die Reform hat einen substanziell positiven Effekt auf das Wirtschaftswachstum, und verschiedene Kennziffern der Armut bzw. Ungleichheit gehen merklich zurück. Schließlich erzeugt sie erhebliche Mehreinnahmen der öffentlichen Hand, die im Jahr 2041 die fiskalischen Kosten erstmals übersteigen.

\section{Fazit}

Die Ergebnisse der Simulationsanalyse verdeutlichen die Vorteile einer beherzten Reform der Sozialabgaben, die den Sonderstatus der Minijobs abschafft und gleichzeitig die Gleitzone bzw. den Übergangsbereich auf 1.800 Euro ausweitet (Reformvariante 2). Eine solche Reform könnte die niedrigen Erwerbseinkommen erheblich entlasten und würde langfristig zu einer starken Ausweitung der Beschäftigung führen. Zudem würden besonders solche Personengruppen von der Reform profitieren, die in der Regel hohe Hürden am Arbeitsmarkt überwinden müssen (geringqualifizierte und alleinerziehende Erwerbspersonen).

Darüber hinaus würden den fiskalischen Kosten der Reform erhebliche fiskalische Mehreinnahmen gegenüberstehen, sodass auf lange Sicht die Tragfähigkeit der öffentlichen Finanzen sogar gestärkt wäre. Eine solche Reform wäre also gut für die Wirtschaft, die Gesellschaft und die öffentlichen Finanzen.

Diese Ergebnisse verdeutlichen, dass die Abschaffung des Sonderstatus des Minijobs, kombiniert mit einer weiteren Ausweitung der Gleitzone, einen wichtigen Beitrag zum Aufbau guter Beschäftigung leisten kann, die sozial abgesichert und fairer entlohnt ist. Damit die simulierten Effekte tatsächlich realisiert werden, sind entschlossene arbeitsmarktpolitische Reformen notwendig. Denn zwei Dinge hat die Corona-Krise offenbart: Ein soziales Sicherungsnetz ist unabdingbar, gerade auch für geringfügig Beschäftigte. Die bestehenden Sonderregelungen sind nur vordergründig von Vorteil, führen aber mittel- und langfristig allzu oft zu dauerhaft geringer Entlohnung, schlechten Aufstiegschancen und geringen Rentenansprüchen. Im Zuge der Reform sollten Minijobs daher nur 
Tabelle 2

Übersicht der Simulationsergebnisse - Reformvariante 2

\begin{tabular}{|c|c|c|c|c|c|c|c|c|c|}
\hline & \multicolumn{3}{|c|}{$\begin{array}{c}\text { Alle } \\
\text { Erwerbspersonen }\end{array}$} & \multicolumn{3}{|c|}{$\begin{array}{l}\text { Geringqualifizierte } \\
\text { Erwerbspersonen }\end{array}$} & \multicolumn{3}{|c|}{$\begin{array}{l}\text { Alleinerziehende } \\
\text { Erwerbspersonen }\end{array}$} \\
\hline & 2020 & 2030 & 2050 & 2020 & 2030 & 2050 & 2020 & 2030 & 2050 \\
\hline BIP (in Mrd. Euro) & 0 & $+7,21$ & $+20,23$ & & & & & & \\
\hline Fiskalische Nettomehreinnahmen (in Mrd. Euro) & $-4,54$ & $-2,26$ & $+2,21$ & & & & & & \\
\hline \multicolumn{10}{|l|}{ Beschäftigung (absolut) } \\
\hline arbeitslos & 0 & -86.700 & -92.600 & 0 & -39.300 & -43.800 & 0 & -20.700 & -25.700 \\
\hline geringfügig beschäftigt & 0 & -77.200 & -76.600 & 0 & -4.700 & -4.100 & 0 & -12.600 & -12.000 \\
\hline teilzeitbeschäftigt & 0 & +159.200 & +162.400 & 0 & +22.900 & +25.300 & 0 & +21.500 & +24.400 \\
\hline vollzeitbeschäftigt & 0 & +4.700 & +6.800 & 0 & +21.100 & +22.600 & 0 & +11.800 & +11.100 \\
\hline Beschäftigung (VZ-Äquivalent) & 0 & +65.000 & +68.900 & 0 & +31.300 & +34.200 & 0 & +19.400 & +22.500 \\
\hline Armutsgefährdungsquote (in Prozentpunkten) & 0 & $-0,11$ & $-0,11$ & 0 & $-1,47$ & $-1,47$ & 0 & $-0,37$ & $-0,40$ \\
\hline Median der Armutsgefährdung (in Euro) & 0 & $+6,65$ & $+6,67$ & 0 & $+68,79$ & $+69,09$ & 0 & $+35,76$ & $+38,51$ \\
\hline Armutsquote (in Prozentpunkten) & 0 & $-0,06$ & $-0,06$ & 0 & $-0,20$ & $-0,20$ & 0 & $-0,30$ & $-0,33$ \\
\hline
\end{tabular}

Anmerkungen: Ergebnis der Modellsimulation. Die Werte geben die jeweiligen Veränderungen gegenüber der Situation seit der Reform vom 1. Juli 2019 wieder. Die Armutsgefährdungsquote ist der Anteil der Erwerbspersonen, die nach Transferzahlungen über weniger als $60 \%$ des Median-Nettoeinkommens verfügen (2019: 15,9\%). Der Median der Armutsgefährdung ist das Median-Nettoeinkommen der armutsgefährdeten Erwerbspersonen. Die Armutsquote ist der Anteil der Erwerbspersonen, die ein Nettoeinkommen unterhalb der Grundsicherung beziehen (2019: 9,6\%).

Quelle: eigene Berechnung.

noch als Hinzuverdienstmöglichkeit für beispielsweise Studierende, Schüler:innen oder Rentner:innen verbleiben. Darüber hinaus sollten Niedriglohnbeschäftigte, von denen viele in Berufen tätig sind, die seit der Corona-Krise als systemrelevant gelten, von den überproportional stark ansteigenden Sozialabgaben entlastet werden, damit sich auch für sie Arbeit finanziell mehr lohnt.

\section{Literatur}

Krebs, T. und M. Scheffel (2015), Quantifizierung der gesamtwirtschaftlichen Effekte ausgewählter Reformvorschläge der Studie „Reforms, investment and growth: an agenda for France, Germany and Europe", Bundesministerium für Wirtschaft und Energie.

Krebs, T. und M. Scheffel (2021), Raus aus der Minijobfalle - Reformen zur Entlastung geringer Einkommen und ihre Auswirkungen auf Beschäftigung, Wachstum und Verteilung sowie die öffentlichen Finanzen, Bertelsmann Stiftung.

Title: The Long-Term Effects of Reforming Social Security to Relieve Low-Income Households

Abstract: In this paper, we use a macroeconomic model to evaluate two reform proposals that, first, abolish the exemption from social security contributions for marginal employment ("mini jobs") and, second, reduce social security contributions for low-income earners. Specifically, the first reform proposal lowers social security contributions for individuals with a gross income of less than 1,300 euro per month. The second reform proposal extends the relief to individuals who earn less than 1,800 euro per month. The main findings show that the second reform proposal leads to substantial relief for low income individuals and incentivises unemployed, marginal employed and parttime employed households to increase their labour supply. While there are already positive shortterm effects on employment, the second reform proposal increases longterm employment by 68,900 full-time equivalent units and the ensuing positive effect on employment and productivity is beneficial for the economy, the society, and for fiscal balances.

JEL Classification: E60, H2O, H6O, J48, J68 